'Departamento de Salud de la Mujer, Escuela de Enfermería, Pontificia Universidad Católica de Chile. Santiago, Chile. ${ }^{2} S$ chool of Social Sciences, Cardiff University, UK

${ }^{3}$ Departamento de Salud de Adulto y Senescente, Escuela de Enfermería, Pontificia Universidad Católica de Chile. Santiago, Chile. ${ }^{4}$ Unidad de Farmacología y Toxicología, Facultad de Medicina, Pontificia Universidad Católica de Chile. Santiago, Chile. ${ }^{5}$ Departamento de Salud Pública,

Escuela de Medicina, Pontificia Universidad Católica de Chile. Santiago, Chile. aEnfermera.

${ }^{b}$ Enfermera-matrona, Magíster en Psicología de la Salud. Pontificia Universidad Católica de Chile. Santiago, Chile.

'Enfermera, Estudiante de Magister en Enfermería, Pontificia Universidad Católica de Chile. Santiago, Chile. dEstadístico. ePhD.

Fuente de apoyo financiero: Fondo Nacional de Desarrollo Científico y Tecnológico (FONDECYT), Proyecto FONDECYT Iniciación en Investigación 11150221. FONDECYT no influyó en el diseño del estudio, en la recolección, análisis o interpretación de los datos ni en la preparación, revisión o aprobación del manuscrito.

Recibido el 11 de junio de 2018, aceptado el 15 de noviembre de 2018.

Correspondencia a: Paulina Bravo Av. Vicuña Mackenna 4860 Macul. Santiago, Chile. pbbravo@uc.cl

\section{Conflicto decisional en personas con diabetes mellitus e hipertensión arterial usuarias del nivel primario de atención de salud de Chile}

\author{
PAULINA BRAVO ${ }^{1,2, \mathrm{a}, \mathrm{e}}$, ANGELINA DOIS $^{3, \mathrm{~b}}$, \\ MARÍA JOSÉ HERNÁNDEZ ${ }^{4, c}$, LUIS VILLARROEL ${ }^{5, \mathrm{~d}, \mathrm{e}}$
}

\section{Decisional conflict among people with diabetes mellitus or hypertension attending primary care}

Background: Decisional conflict refers to the personal uncertainty about which course of action to take when the choice involves risk, regret, or challenge to personal life values. Aim: To determine the level of decisional conflict (DC) of people with Diabetes Mellitus (DM) or High Blood Pressure (HBP) attending primary care centers (PCC) in Chile. Patients and Methods: A Spanish version of the Decisional Conflict Scale (DCS) was applied to patients who were recruited if they had DM or HBP, were 18 years old or older, and had an appointment at the PCC the day of the recruitment. The scale was self-administered. Analysis of covariance (ANCOVA) was used to determine association between DC and other variables of interest while controlling confounding variables. Results: The scale was answered by 1075 participants from 24 PCC aged $62 \pm 14$ years (74\% female). Average score for the DCS scale was $16.8 \pm 12.9$ of a maximum of 100 points indicating a higher DC. The sub-scale "information" had the highest score (19.9 \pm 20.0$)$. Low educational level and older age were significantly associated with higher DCS scores $(p<0.05)$. Having a bad health perception, deciding to initiate a medical treatment and being attended by a doctor were significantly associated with higher DC. These associations persisted when confounding variables such as sex, age and education were controlled. Conclusions: People with $D M$ or HBP who have a poor health perception, who initiated their treatment and were attended by a doctor had higher levels of DC, independent of their age and educational level.

(Rev Med Chile 2018; 146: 1286-1293)

Key words: Chronic Disease; Decision Making; Primary Health Care.

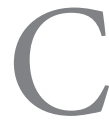

hile ha experimentado un rápido cambio epidemiológico, con un aumento progresivo de enfermedades crónicas no transmisibles (ECNT). De acuerdo a la última Encuesta Nacional de Salud, la prevalencia de sospecha de Hipertensión Arterial (HTA) alcanza a 27,6\% de la población, porcentaje que llega hasta $73 \%$ en adultos mayores. Adicionalmente, la sospecha de Diabetes Mellitus (DM) afecta a 12,3\% de la población, aumentando también con la edad ${ }^{1}$.

Este nuevo panorama epidemiológico supone la necesidad de involucrar a las personas activamente en el cuidado de su salud. Ayudar a que las personas participen en las decisiones clínicas 
podría contribuir al aumento de la adherencia a los tratamientos y consecuentemente a mejores resultados clínicos ${ }^{2,3}$. En este sentido, se espera que personas más involucradas comprendan mejor las razones que sustentan estas decisiones y presenten bajos niveles de conflicto decisional (CD $)^{4}$. El CD se ha definido como la incertidumbre sentida por las personas cuando enfrentan una decisión que involucra riesgo, pérdida, arrepentimiento o desafíos a los valores de la persona ${ }^{5}$, lo que podría llevarlo a aceptar o rechazar el curso de acción determinado ${ }^{6}$.

Aún cuando en Chile existe interés por promover la participación de las personas ${ }^{7,8}$, no existen reportes que permitan identificar qué tanto $\mathrm{CD}$ enfrentan las personas con ECNT.

El objetivo de este artículo es determinar el nivel de CD de personas con DM y/o HTA usuarias de Centros de Salud Familiar de Chile.

\section{Material y Método}

Se realizó un estudio de corte transversal, observacional e individual con usuarios de 24 CESFAM de la zona norte, centro y sur de Chile, entre enero y julio de 2017. El estudio fue aprobado por el Comité Ético Científico de la Facultad de Medicina de la Pontificia Universidad Católica de Chile (número: 15-310) y de acuerdo a la legislación vigente contó con las autorizaciones locales correspondientes.

Los participantes se seleccionaron a través de muestreo intencionado del universo conformado por el total de usuarios que recibieron atención de salud el día de recolección de la información y que cumplieran con los criterios de inclusión: ser mayor de 18 años, tener diagnóstico de HTA y/o DM y estar en capacidad para consentir. Todos los participantes firmaron un documento de consentimiento informado.

\section{Recolección de la información}

Los participantes auto-completaron de manera individual (o asistida según necesidad) un set de cuestionarios que incluía datos sociodemográficos, percepción del estado de salud, información sobre la decisión clínica tomada durante la atención con el profesional de la salud (tipo de decisión y profesional que lo atendió) y la Escala de Conflicto Decisional (ECD). La ECD recoge información sobre cinco dimensiones: incerti- dumbre, información, clarificación de valores, apoyo percibido y efectividad de la decisión, en una escala de Likert de 5 puntos $^{9}$ (Tabla 1). Los resultados pueden variar de 0 a 100 , mientras más alto el resultado mayor $\mathrm{CD}$, y puntajes mayores a 25 puntos se asocian a menores probabilidades de implementar la decisión y la efectividad de esta ${ }^{10}$. $\mathrm{El}$ instrumento fue originalmente desarrollado en Canadá y validado para la población chilena con estudiantes universitarios reportando un Alpha de Cronbach de $0,80^{6}$. Este estudio utilizó por primera vez la ECD en usuarios de atención primaria de salud (APS) y dado que estos usuarios tienen características sociodemográficas distintas a la muestra en la que la ECD fue validada, el equipo de investigación realizó un análisis de la confiabilidad del instrumento y se obtuvo un Alpha de Cronbach de 0,88.

\section{Tabla 1. Escala de Conflicto Decisional}

\section{Pregunta}

1. Esta es una decisión fácil de tomar para mí

2. Estoy seguro de lo que tengo que hacer en esta decisión

3. Está claro cuál es la mejor opción para mí

4. Estoy consciente de las diferentes opciones que tengo en esta decisión

5. Siento que conozco los aspectos positivos de cada opción

6. Siento que conozco los aspectos negativos de cada opción

7. Tengo claridad acerca de cuán importantes son los aspectos positivos para mí

8. Tengo claridad acerca de cuán importantes son los aspectos negativos para mí

9. Tengo claridad acerca de qué es lo más importante para mí (los aspectos positivos o los aspectos negativos)

10. Estoy tomando esta decisión sin ninguna presión de otras personas

11. Tengo la cantidad apropiada de apoyo de otros para tomar esta decisión

12. Cuento con suficientes consejos acerca de las opciones

13. Siento que he hecho una elección bien informada

14. Mi decisión muestra lo que es importante para mí

15. Espero mantenerme firme en mi decisión

16. Estoy satisfecho con mi decisión 


\section{Análisis de la información}

Las variables categóricas se presentan como número de casos y porcentajes y las variables numéricas se presentan con promedio \pm desviación estándar y los scores se presentan además con cuartiles (percentiles 25, 50 y 75).

Para la comparación de porcentajes se usó el test $\chi^{2}$ de Pearson. Para comparar promedios entre dos grupos se usó el test t de Student para muestras independientes y para comparar promedios de scores se usó el test no paramétrico de rangos de Wilcoxon. Para comparar más de dos promedios se usó análisis de la varianza (ANOVA) en una vía, además del test de Hochberg para comparaciones post hoc. Para determinar la asociación de ECD con otras variables de interés controlando el efecto de sexo, edad y nivel educacional se ajustaron modelos de análisis de la covarianza.

Se consideró significativo valores $\mathrm{p}$ inferiores o iguales a 0,05. Los análisis se hicieron usando el programa estadístico SPSS 17.

\section{Resultados}

\section{Participantes}

La muestra final fue de 1.075 usuarios. La mayoría eran mujeres (74\%), con una edad promedio de 61,6 años $( \pm 13,6)$. El 12,7\% de los participantes tenía diagnóstico de DM, 52,1\% HTA y 35,2\% ambas patologías. La Tabla 2 resume las principales características de este grupo.

\section{Conflicto decisional}

El promedio de score para la ECD fue de 16,8 $( \pm 12,9)$. La subescala con mayor puntaje fue Información $(19,9 \pm 20)$. La subescala con menor puntuación fue Efectividad de la Decisión $(11,1 \pm 12,5)$. La Tabla 3 detalla los puntajes para cada subescala.

El análisis incluyó la determinación de percentiles, y se consideró que valores mayores o iguales al percentil 75 corresponden a alto $\mathrm{CD}$, lo que para la escala global es equivalente a 25 puntos (Tabla 3 ), que coincide con lo reportado en la literatura como puntaje asociado a menor implementación de la decisión.

\section{Indicadores de alto conflicto decisional}

El nivel de CD según sexo no presentó diferencias significativas para la ECD y en ninguna de las subescalas (Tabla 4). La ECD no presentó diferencias significativas por grupo etario. Sin embargo, las personas de 65 años o más tienen puntajes significativamente mayores para las subescalas de Información, Valores y Apoyo $(\mathrm{p}<0,001)$. Solo para la subescala Incertidum-

\section{Tabla 2. Características de los participantes}

\begin{tabular}{|c|c|c|}
\hline & $\mathbf{n}$ & $\%$ \\
\hline \multicolumn{3}{|l|}{ Sexo } \\
\hline Mujeres & 795 & 74 \\
\hline Hombres & 280 & 26 \\
\hline \multicolumn{3}{|l|}{ Nacionalidad } \\
\hline Chileno & 1.054 & 98 \\
\hline Extranjero & 21 & 2 \\
\hline \multicolumn{3}{|l|}{ Nivel educacional } \\
\hline Básica (completa e incompleta) & 415 & 38,6 \\
\hline Media (completa e incompleta) & 477 & 44,3 \\
\hline Superior (completa e incompleta) & 148 & 13,8 \\
\hline Sin estudios & 34 & 3,2 \\
\hline \multicolumn{3}{|l|}{ Ocupación } \\
\hline Trabajador dependiente & 219 & 20,4 \\
\hline Trabajador independiente & 101 & 9,4 \\
\hline Dueña de casa & 265 & 24,7 \\
\hline Cesante & 44 & 4,1 \\
\hline Pensionado & 439 & 40,8 \\
\hline Estudiante & 7 & 0,7 \\
\hline \multicolumn{3}{|l|}{ Enfermedades crónicas } \\
\hline Diabetes Mellitus (DM) & 137 & 12,7 \\
\hline Hipertensión Arterial (HTA) & 561 & 52,2 \\
\hline $\mathrm{DM}+\mathrm{HTA}$ & 377 & 35,1 \\
\hline \multicolumn{3}{|l|}{ Frecuencia que asiste al centro de salud } \\
\hline Primera atención de salud & 14 & 1,3 \\
\hline Al menos 1 vez por mes & 435 & 40,5 \\
\hline Cada 2-6 meses & 584 & 54,3 \\
\hline 1 vez por año o más & 42 & 3,2 \\
\hline \multicolumn{3}{|c|}{ Profesional que lo atendió por última vez } \\
\hline Enfermera & 161 & 15 \\
\hline Médico & 571 & 53,1 \\
\hline Matrona & 61 & 5,7 \\
\hline Psicólogo & 43 & 4 \\
\hline Dentista & 38 & 3,5 \\
\hline Kinesiólogo & 74 & 6,9 \\
\hline Nutricionista & 85 & 7,9 \\
\hline Otro & 42 & 3,9 \\
\hline \multicolumn{3}{|l|}{ Percepción del estado de salud } \\
\hline Excelente & 30 & 2,8 \\
\hline Muy buena & 51 & 4,7 \\
\hline Buena & 405 & 37,7 \\
\hline Regular & 470 & 43,7 \\
\hline Mala & 119 & 11,1 \\
\hline
\end{tabular}


Tabla 3. Puntajes obtenidos para ECD por promedio y percentil

\begin{tabular}{|c|c|c|c|c|c|c|}
\hline & \multirow[t]{2}{*}{$\mathbf{n}$} & \multirow{2}{*}{$\begin{array}{l}\text { Promedio } \\
\text { ( } \pm \text { SD) }\end{array}$} & \multirow{2}{*}{$\begin{array}{c}\text { Puntaje máximo } \\
\text { obtenible* }\end{array}$} & \multicolumn{3}{|c|}{ Percentiles } \\
\hline & & & & 25 & $\mathbf{5 0}$ & 75 \\
\hline Conflicto decisional global & 1.071 & $16,8 \pm 12,9$ & 100 & 4,6 & 15,6 & 25,0 \\
\hline Subescala información & 1.075 & $19,9 \pm 20,0$ & 100 & 0,0 & 25,0 & 25,0 \\
\hline Subescala valores & 1.075 & $17,4 \pm 20,0$ & 100 & 0,0 & 16,6 & 25,0 \\
\hline Subescala apoyo & 1.075 & $19,7 \pm 19,2$ & 100 & 0,0 & 16,6 & 33,3 \\
\hline Subescala incertidumbre & 1.075 & $17,6 \pm 18,9$ & 100 & 0,0 & 16,6 & 25,0 \\
\hline Subescala efectividad & 1.071 & $11,1 \pm 12,5$ & 100 & 0,0 & 6,2 & 25,0 \\
\hline
\end{tabular}

${ }^{*}$ A mayor puntaje obtenido, mayor CD.

Tabla 4. Puntajes obtenidos para ECD según sexo

\begin{tabular}{|lccc|}
\hline & $\begin{array}{c}\text { Mujeres } \\
(\mathbf{n}=\mathbf{7 9 5})\end{array}$ & $\begin{array}{c}\text { Hombres } \\
(\mathbf{n}=\mathbf{2 8 0})\end{array}$ & $\begin{array}{c}\text { Valor } \\
\text { p }\end{array}$ \\
\hline Conflicto decisional global & $16,8 \pm 13,0$ & $16,7 \pm 12,7$ & 0,919 \\
\hline Sub-escala información & $19,2 \pm 20,0$ & $21,6 \pm 20,1$ & 0,065 \\
\hline Sub-escala valores & $17,2 \pm 20,2$ & $18,1 \pm 19,1$ & 0,211 \\
\hline Sub-escala apoyo & $20,5 \pm 19,9$ & $17,7 \pm 16,8$ & 0,107 \\
\hline Sub-escala incertidumbre & $17,8 \pm 19,3$ & $16,9 \pm 17,6$ & 0,774 \\
\hline Sub-escala efectividad & $11,1 \pm 12,6$ & $11,3 \pm 12,4$ & 0,669 \\
\hline
\end{tabular}

Tabla 5. Puntajes obtenidos para ECD según nivel educacional

\begin{tabular}{|lccccc|}
\hline & $\begin{array}{c}\text { Educación } \\
\text { básica } \\
\mathbf{( n = 4 1 5 )}\end{array}$ & $\begin{array}{c}\text { Educación } \\
\text { media } \\
(\mathbf{n}=\mathbf{4 7 7})\end{array}$ & $\begin{array}{c}\text { Educación } \\
\text { superior } \\
\mathbf{( n = 1 4 8 )}\end{array}$ & $\begin{array}{c}\text { Sin } \\
\text { educación } \\
\mathbf{( n = 3 4 )}\end{array}$ & $\begin{array}{c}\text { Valor } \\
\mathbf{p}\end{array}$ \\
\hline Conflicto decisional global & $18,5 \pm 13,2^{\dagger}$ & $16,1 \pm 12,5$ & $14,4 \pm 13,6$ & $17,6 \pm 11,2$ & 0,004 \\
\hline Sub-escala información & $23,1 \pm 21,6^{\dagger}$ & $18,5 \pm 18,3$ & $16,0 \pm 20,3$ & $18,1 \pm 16,9$ & $<0,001$ \\
\hline Sub-escala valores & $20,2 \pm 21,3^{\dagger}$ & $16,1 \pm 18,6$ & $13,6 \pm 20,2$ & $18,3 \pm 16,5$ & 0,001 \\
\hline Sub-escala apoyo & $20,9 \pm 19,3$ & $19,5 \pm 19,0$ & $16,9 \pm 19,7$ & $22,3 \pm 17,1$ & 0,154 \\
\hline Sub-escala incertidumbre & $18,7 \pm 18,8$ & $17,0 \pm 18,9$ & $17,0 \pm 20,3$ & $15,4 \pm 13,7$ & 0,486 \\
\hline Sub-escala efectividad & $11,6 \pm 12,3$ & $11,0 \pm 12,4$ & $9,4 \pm 13,1$ & $14,8 \pm 13,4$ & 0,096 \\
\hline
\end{tabular}

†Diferencia significativa para ese grupo.

bre las personas menores de 50 años presentaron puntajes significativamente mayores (promedio $19,9 \pm 20,2, \mathrm{p}<0,05)$.

El nivel educacional se asoció a mayores puntajes de CD. Así, las personas que indicaron tener educación básica (completa e incompleta) tendieron a presentar un score global mayor $(\mathrm{p}=0,004), \mathrm{y}$ reportar puntajes más altos para las subescalas de Información y Valores (Tabla 5). Por otro lado, la frecuencia con la que los usuarios asistían al centro de salud no se asoció al puntaje de CD. Sin embargo, la percepción del estado de salud mostró puntajes significativamente mayores para los participantes con estado de salud malo o regular (Tabla 6). Adicionalmente, la proporción de personas con puntajes iguales o mayores a 25 
Tabla 6. Puntajes obtenidos para ECD según percepción de salud

\begin{tabular}{|lcccccc|}
\hline & $\begin{array}{c}\text { Excelente } \\
(\mathbf{n}=\mathbf{3 0})\end{array}$ & $\begin{array}{c}\text { Muy buena } \\
(\mathbf{n}=\mathbf{5 1})\end{array}$ & $\begin{array}{c}\text { Buena } \\
(\mathbf{n}=\mathbf{4 0 5})\end{array}$ & $\begin{array}{c}\text { Regular } \\
(\mathbf{n}=\mathbf{4 7 0})\end{array}$ & $\begin{array}{c}\text { Mala } \\
(\mathbf{n}=\mathbf{1 1 9})\end{array}$ & Valor $\mathbf{p}$ \\
\hline Conflicto decisional global & $11,3 \pm 11,9$ & $10,4 \pm 10,2$ & $15,2 \pm 11,8$ & $18,6 \pm 13,6^{\dagger}$ & $19,2 \pm 13,6^{\dagger}$ & $<0,001$ \\
\hline
\end{tabular}

${ }^{\dagger}$ Diferencias significativas para esos grupos.

Tabla 7. Puntajes obtenidos para ECD según tipo de decisión realizada

\begin{tabular}{|lcccccc|}
\hline & $\begin{array}{c}\text { Inicio } \\
\text { tratamiento } \\
(\mathbf{n}=\mathbf{2 2 3})\end{array}$ & $\begin{array}{c}\text { Continuación } \\
\text { tratamiento } \\
(\mathbf{n}=\mathbf{5 0 2})\end{array}$ & $\begin{array}{c}\text { Toma } \\
\text { exámenes } \\
(\mathbf{n = 2 0 7})\end{array}$ & $\begin{array}{c}\text { Cambios } \\
\text { alimentación } \\
(\mathbf{n}=\mathbf{6 0})\end{array}$ & $\begin{array}{c}\text { Aumentar } \\
\text { ejercicios } \\
(\mathbf{n}=\mathbf{2} \text { 9) }\end{array}$ & $\begin{array}{c}\text { Valor } \\
\mathbf{p}\end{array}$ \\
\hline Conflicto decisional global & $19,0 \pm 13,4$ & $16,1 \pm 13,2$ & $16,0 \pm 12,4$ & $17,7 \pm 11,4$ & $17,0 \pm 11,0$ & 0,066 \\
\hline Subescala información & $24,4 \pm 21,0^{\dagger}$ & $19,3 \pm 20,6$ & $18,0 \pm 17,9$ & $18,5 \pm 18,7$ & $18,3 \pm 17,7$ & 0,009 \\
\hline Subescala incertidumbre & $18,7 \pm 19,0$ & $16,5 \pm 19,2$ & $15,4 \pm 16,3$ & $29,0 \pm 21,1^{\dagger}$ & $17,2 \pm 17,3$ & $<0,001$ \\
\hline
\end{tabular}

${ }^{\dagger}$ Diferencia significativa para ese grupo.

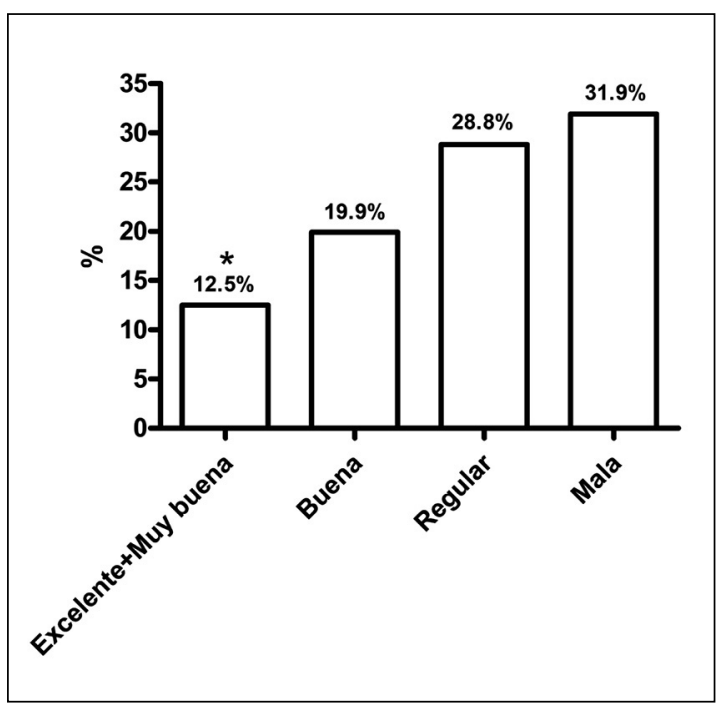

Figura 1. Conflicto decisional $>25$ puntos según percepción de salud.

puntos (CD alto) es significativamente menor para aquellos que reportaron un estado de salud excelente o muy bueno respecto a aquellos con una mala o regular percepción de salud (Figura 1). De acuerdo al tipo de decisión realizada durante la consulta iniciar tratamiento y hacer cambios en la alimentación obtuvieron puntajes significativamente más altos para las subescalas de Infor-

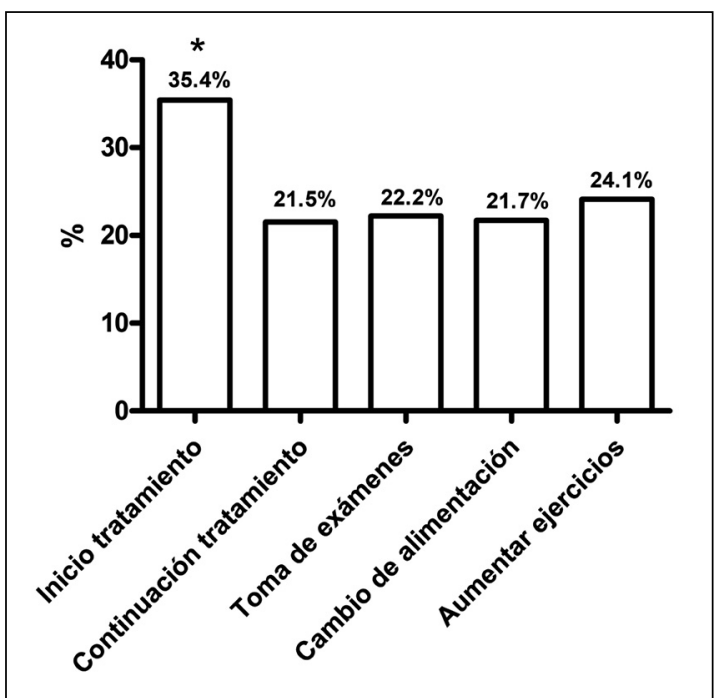

Figura 2. Conflicto decisional $>25$ puntos según percepción de salud.

mación e Incertidumbre, respectivamente (Tabla 7). Interesantemente, las proporción de personas con alto CD fue significativamente mayor si decidieron iniciar tratamiento $(\mathrm{p}<0,05)$ (Figura 2). Adicionalmente, cuando fueron médicos quienes brindaron atención sanitaria el puntaje global para la ECD fue más alto que el obtenido con la atención de otros profesionales (Figura 3 ). 


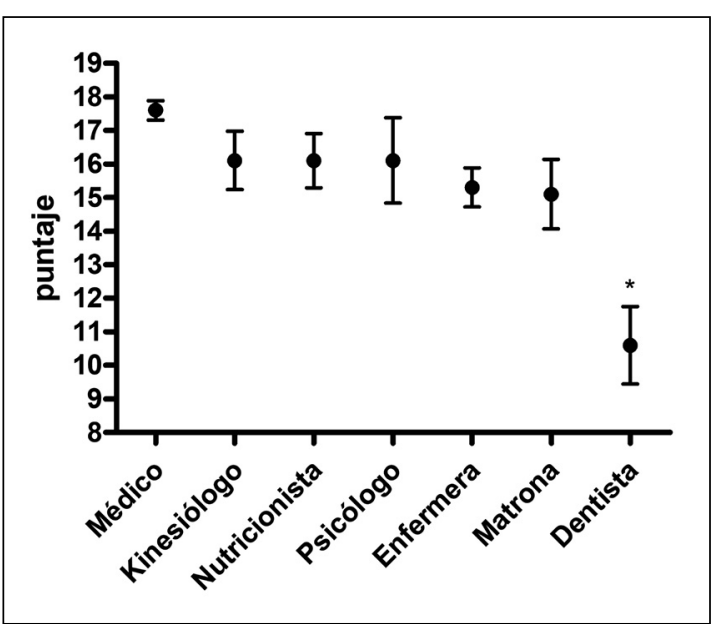

Figura 3. Puntajes obtenidos para ECD según profesional de salud (promedio \pm error estándar).

Finalmente, al ajustar modelos de análisis de la covarianza para explicar el puntaje de la ECD en función de las variables percepción de salud, profesional que lo atendió y tipo de decisión, y controlando el efecto de sexo, nivel educacional y edad, estas se mantuvieron significativamente asociadas a mayores puntajes de la escala.

\section{Discusión}

Este estudio determinó los niveles de CD para las dos enfermedades crónicas más prevalentes en Chile, encontrándose niveles de conflicto similares en hombres y mujeres, pero asociado de manera significativa a la percepción de salud, tipo de decisión realizada y profesional que lo atendió. La evidencia da cuenta que entre los factores modificables asociados al CD se puede distinguir: a) conocimientos inadecuados respecto de las opciones de tratamiento y los daños/ beneficios asociados a cada una; b) falta de apoyo para la toma de decisiones y c) la percepción de no poder tomar una decisión de buena calidad ${ }^{5,11}$. Los resultados de este estudio son similares a los reportados por otros investigadores respecto de los dos primeros, sin embargo, la efectividad de la decisión fue la subescala que presentó el menor $\mathrm{CD}$. Al analizar cada una de las subescalas el mayor CD se presentó en el área de la Información. Esta es específicamente considerada uno de los desafíos a la hora de enfrentar una decisión en salud, ya que en la medida que los usuarios están mejor informados acerca de su enfermedad y de las opciones de tratamiento disponibles se encontrarán en mejores condiciones para decidir lo que es mejor para ellos $^{11,12}$. Sin embargo, no basta con la mera entrega de la información ${ }^{13}$ sino que es necesario que el profesional establezca una relación empática y de colaboración con el usuario que le permita sentirse acompañado en el proceso de decidir, como también que utilice las herramientas necesarias para que la información pueda ser comprendida por ellos en función de su edad y nivel educacional ${ }^{14,15}$, variables que en este estudio aparecen asociadas a la presencia de CD. Esto es especialmente relevante en el contexto de enfermedades crónicas, ya que ha sido reportado que el CD se asocia de manera significativa al arrepentimiento de la decisión ${ }^{16}$, hecho que podría afectar la adherencia y por tanto comprometer los resultados clínicos y calidad de vida de los usuarios ${ }^{17}$.

Los hallazgos de los modelos de covarianza realizados para percepción de salud, profesional que lo atendió y tipo de decisión, indican que el efecto de estas variables no está mediado por sexo, edad o nivel educacional. Vale decir, la relación que hay entre estas variables y el nivel CD no es modificada, independiente del sexo, edad y educación de la persona. De ello se desprende que la mejor opción terapéutica no puede seleccionarse solo a partir de los datos objetivos y la evidencia disponible, sino que deben considerarse aspectos relevantes para el usuario como sus valores y preferencias, el contexto personal y familiar en el que se desarrolla, que forman parte del mundo subjetivo y que dan significado a la experiencia de enfermar de esa persona en particular ${ }^{12,18}$. En esa línea, el uso de herramientas para la toma de decisiones compartidas que combinen la mejor evidencia disponible con las preferencias y el contexto del usuario ${ }^{14,19}$ pueden considerarse como un elemento terapéutico que reduzca la posibilidad de abandono del tratamiento. Bailey et al demostraron que el uso de estas herramientas reduce de manera significativa el CD de personas con $\mathrm{DM}^{10}$.

Si bien algunos estudios muestran que el número de visitas a un centro de salud crece al aumentar el $\mathrm{CD}^{5}$, esta no fue una característica de los participantes en esta investigación, lo que podría explicarse a partir de la experiencia que han tenido con los profesionales que les atienden 
y cómo estos han podido ayudarlos a tomar una decisión, como también debido al modelo de relación clásicamente paternalista con el que se ha desarrollado la atención de salud, que instala al usuario en una posición jerárquicamente inferior al profesional de la salud lo que limita la posibilidad de participar en las decisiones relativas a su salud $^{8,20}$. Referente a este tema, es importante recordar que la participación surge como imperativo ético ${ }^{21}$ para mejorar las condiciones de vida de la población, al reconocer su rol activo y central en la mantención y recuperación de la salud. Es así que la Organización Mundial de la Salud (OMS) ${ }^{22}$ la considera como una función esencial de la salud pública. Sin embargo, esta ha estado más presente en acciones con base comunitaria que en acciones terapéuticas propias de la restauración de la salud, como lo es el encuentro clínico.

El Modelo de Atención Integral en Salud Familiar y Comunitaria vigente define la atención de salud como un proceso integral y continuo cuyo foco son las necesidades de salud de las personas a partir del desarrollo de competencias de autocuidado $^{23}$, por lo que reconocer el CD e intervenir para mejorar la participación en salud pareciera ser un ejercicio clave para hacer eco al llamado de la OMS de fortalecer la APS $^{24}$, sobre todo en países como Chile que observan un aumento sostenido de la esperanza de vida y de la prevalencia de ECNT en una población que se atiende mayoritariamente en el sistema público de salud ${ }^{25}$.

La principal limitación de este estudio podría referirse al momento en que se realizó la recolección de los datos. Los participantes fueron contactados inmediatamente a la salida de la consulta con el profesional de la salud, con el fin de evitar sesgo de memoria en relación a la decisión tomada en dicho encuentro. Sin embargo, esto podría haber afectado el reporte de CD, en cuanto los participantes pueden haber reportado menores niveles de CD con el fin de no afectar su relación con el profesional de salud, resultando en una respuesta socialmente deseada, tal como ha sido reportado en otras investigaciones ${ }^{16}$.

La fortaleza de este estudio es entregar por primera vez la medición de CD en esta población, utilizando un instrumento validado y ampliamente utilizado a nivel internacional. Esto podría contribuir al desarrollo de intervenciones que concentren sus esfuerzos en apoyar a aquellos que, pudiendo tener un mayor conflicto decisional, puedan tener dificultades al momento de elegir alguna acción terapéutica y por lo tanto adherir a ella. Adicionalmente, este estudio aporta con datos empíricos al establecimiento del puntaje de corte de la ECD en 25 puntos para considerar un alto nivel de $\mathrm{CD}$, elemento que podría contribuir a diagnosticar necesidades relativas al CD y guiar intervenciones para apoyar la resolución del mismo. Futuras investigaciones podrían considerar ampliar la medición de CD a otros grupos, como usuarios sin patologías crónicas, para con ello comprender de mejor manera cómo se está involucrando a los usuarios en la toma de decisiones clínicas y cuánto están comprendiendo acerca de ellas.

\section{Referencias}

1. Ministerio de Salud MINSAL. Encuesta Nacional de Salud 2016-2017 Primeros resultados 2018 [citado el 21 de mayo de 2018]. Available from: http://www.minsal. cl/wp-content/uploads/2017/11/ENS-2016-17_PRIMEROS-RESULTADOS.pdf.

2. Stacey D, Legare F, Lewis K, Barry MJ, Bennett CL, Eden $\mathrm{KB}$, et al. Decision aids for people facing health treatment or screening decisions. The Cochrane database of systematic reviews. 2017;4: Cd001431.

3. Knops AM, Legemate DA, Goossens A, Bossuyt PM, Ubbink DT. Decision aids for patients facing a surgical treatment decision: a systematic review and meta-analysis. Annals of surgery. 2013; 257 (5): 860-6.

4. Holzel LP, Kriston L, Harter M. Patient preference for involvement, experienced involvement, decisional conflict, and satisfaction with physician: a structural equation model test. BMC health services research 2013; 13: 231.

5. Thompson-Leduc $P$, Turcotte $S$, Labrecque $M$, Légaré F. Prevalence of clinically significant decisional conflict: an analysis of five studies on decision-making in primary care. BMJ Open 2016; 6 (6).

6. Urrutia M, Campos S, O'Connor A. [Validation of a Spanish version of the Decisional Conflict scale]. Rev Med Chile 2008; 136 (11): 1439-47.

7. Ministerio de Salud de Chile MINSAL. Estrategia nacional de salud para el cumplimiento de los objetivos sanitarios de la década 2011-2020. Chile 2010.

8. Bravo P, Dois A, Cabieses B, Bustamante C, Campos S, Stacey D. Patient-centred care and shared decision making in Chile: Rising momentum for progress and implementation in clinical practice. Zeitschrift fur Evi- 
denz, Fortbildung und Qualitat im Gesundheitswesen. 2017; 123-4: 28-31.

9. O'Connor AM. Validation of a decisional conflict scale. Medical decision making: an international journal of the Society for Medical Decision Making 1995; 15 (1): 25-30.

10. Bailey RA, Pfeifer M, Shillington AC, Harshaw Q, Funnell MM, VanWingen J, et al. Effect of a patient decision aid (PDA) for type 2 diabetes on knowledge, decisional self-efficacy, and decisional conflict. BMC health services research 2016; 16: 10.

11. Legare F, Leblanc A, Robitaille H, Turcotte S. The decisional conflict scale: moving from the individual to the dyad level. Zeitschrift fur Evidenz, Fortbildung und Qualitat im Gesundheitswesen 2012; 106 (4): 247-52.

12. Serrano V, Larrea-Mantilla L, Rodríguez-Gutiérrez R, Spencer-Bonilla G, Malaga G, Hargraves I, et al. [Shared decision making in patients with diabetes mellitus]. Rev Med Chile 2017; 145 (5): 641-9.

13. Joseph-Williams N, Elwyn G, Edwards A. Knowledge is not power for patients: a systematic review and thematic synthesis of patient-reported barriers and facilitators to shared decision making. Patient Educ Couns 2014; 94 (3): 291-309.

14. Bravo P, Contreras A, Perestelo-Pérez L, Pérez-Ramos J, Málaga G. En busca de una salud más participativa: compartiendo decisiones de salud. Rev Peru Med Exp Salud Publica 2013; 30 (4): 691-7.

15. Bravo P, Edwards A, Barr P, Scholl I, Elwyn G, McAllister M. Conceptualising patient empowerment: a mixed methods study. BMC health services research. 2015; In press.

16. Becerra-Pérez MM, Menear M, Turcotte $S$, Labrecque M, Legare F. More primary care patients regret health decisions if they experienced decisional conflict in the consultation: a secondary analysis of a multicenter descriptive study. BMC family practice 2016; 17 (1): 156.

17. Montori V, Gafni A, Charles C. A shared treatment decision-making approach betwee patients with chronic conditions and their clinicians: the case of diabetes. Health Expectations 2006; 9: 25-36.

18. Makoul G, Clayman ML. An integrative model of shared decision making in medical encounters. Patient Educ Couns 2006; 60 (3): 301-12.

19. Elwyn G, Kreuwel I, Durand MA, Sivell S, Joseph-Williams N, Evans R, et al. How to develop web-based decision support interventions for patients: a process map. Patient Educ Couns 2011; 82 (2): 260-5.

20. Joseph-Williams N, Edwards A, Elwyn G. Power imbalance prevents shared decision making. BMJ 2014; 348: g3178.

21. Elwyn G, Tilburt L, Montori V. The ethical imperative for shared decision-making. European Journal for Person Centered Healthcare 2013; 1 (1): 129-31.

22. Organización Panamericana de la Salud OPS. La salud pública en las Americas: nuevos conceptos, analisis del desempeño y bases para la acción. In: OPS, editor. Washington 2002.

23. Bass C. Modelo de salud familiar en Chile y mayor resolutividad de la atención primaria de salud: ¿contradictorios o complementarios? Medwave 2012; 12 (11): e5571.

24. Ase I, Burijovich J. La estrategia de Atención Primaria de la Salud: ¿progresividad o regresividad en el derecho a la salud? Salud Colect 2009; 5 (1): 27-47.

25. Aravena LP, Inostroza PM. ¿Salud Pública o Privada? Los factores más importantes al evaluar el sistema de salud en Chile. Rev Med Chile 2015; 143: 244-51. 